\title{
Application of Invasive Weed Optimization in Optimizing the Allocation of WMS Rack
}

\author{
Yue Duan ${ }^{1, a, *}$, Linli Zhou ${ }^{2, b}$ and Yue $\mathrm{Wu}^{2, \mathrm{c}}$ \\ ${ }^{1}$ Institute of Intelligent Machines, Chinese Academy of Sciences, Hefei 230031, China. \\ adyue0927@163.com, blinlizhou@iim.ac.cn, 'wuyue@iim.ac.cn
}

Keywords: WMS (warehouse management system), Multi-objective optimization model, IWO (invasive weed optimization).

\begin{abstract}
In recent years, with the development of modern manufacturing and logistics industry, the development of warehousing management has become more and more rapid, and the status of the logistics industry is getting higher and higher. Therefore, the requirements for warehousing management are getting higher and higher. In this paper, the problem of the allocation strategy of the warehouse in the automated warehouse is discussed, and the optimization model of the multi - target location allocation is established to improve the efficiency of the warehousing and keep the shelf stability and the similar neighbor storage as the optimization target. Then the weighting coefficient method is used to transform the multi - objective optimization model into a single-objective optimization model. Finally, the IWO (invasive weed optimization) is used to solve the single target optimization model, and the simulation experiment is carried out using Matlab software to get the new layout. It can be seen from the simulation results that the results show that IWO can effectively solve the problem of optimal allocation of cargo warehouse
\end{abstract}

\section{Introduction}

The WMS (Warehouse Management System) is a modern warehouse storage system that has a wide range of applications for item storage, so the efficiency of a warehouse is critical. The level of efficiency of an automated warehouse depends mainly on the allocation of reservoir and cargo location strategy. In this paper, an invasive weed algorithm is proposed to solve the problem of cargo location optimization. Through the establishment of mathematical model and simulation using matlab, the simulation results show that the weed algorithm can effectively solve the problem of the optimal allocation of the warehouse.

\section{Introduction of Invasive Weed Optimization}

Invasive Weed Optimization (IWO) is a numerical optimization algorithm proposed by A.R. Mehrabian and C. Lucas in the University of Tehran in $2006^{[1]}$. The algorithm simulates the process of reproduction of weeds in nature with strong vitality and strong adaptability to the environment. The survival and reproduction of plants in nature have $\mathrm{r}$ choice and $\mathrm{k}$ choice in two ways. $\mathrm{R}$ choice refers to the unstable and unpredictable environment to choose fast growing, fast breeding and fasting plants. K choice means that in a stable and predictable environment, the growth of slow, slow propagation, slow death of plants with greater competitiveness. IWO algorithm is simple in structure, with less parameters, fast speed, good robustness, taking into account the global search and local search, with a large potential for engineering applications ${ }^{[2,3]}$.

The basic steps for IWO applications are as follows:

1. Determine the algorithm parameters. Including the number of initial weeds $N_{0}$, the maximum number of iter $_{\max }$, the maximum weed number $P_{\max }$, the maximum seed number $S_{\max }$ and the minimum seed number $S_{\text {min }}$, the non-linear modulation coefficient n, the initial standard deviation of weed diffusion $\sigma_{\text {initial }}$ and the final standard deviation $\sigma_{\text {final }}$. 
2. Initialize the weed position. The initial solution of $N_{0}$ problems is generated randomly.

3. Weed breeding. The number of seeds produced per weed is calculated from the fitness function values of each weed. Wells with high fitness values produce more seeds, and the number of seeds produced per weed is linearly related to the fitness value. The formula is as follows:

$$
N_{\text {seed }}=\frac{F-F_{\text {worst }}}{F_{\text {best }}-F_{\text {worst }}} \times\left(S_{\max }-S_{\min }\right)+S_{\text {min }}
$$

$N_{\text {seed }}$ is the number of seeds produced by the current weeds, $\mathrm{F}$ is the fitness value of the current weeds, $F_{\text {best }}$ and $F_{\text {worse }}$ are the best and worst weed in the current weed population.

4. Space diffusion. The seeds produced by the parent weeds were randomly spread around the parent weeds in a normal distribution. And the standard deviation values are changed as follows:

$$
\sigma_{\text {iter }}=\frac{\left(\text { iter }_{\max }-\text { iter }^{n}\right.}{\text { iter }_{\max }^{n}}\left(\sigma_{\text {initial }}-\sigma_{\text {final }}\right)+\sigma_{\text {final }}
$$

In equation (2), iter is the current number of iterations, and $\sigma_{\text {iter }}$ is the standard deviation used for the current iteration. The above equation shows that, at the beginning of the iteration, the standard deviation is large and the algorithm is expressed as a global search. With the increase of the number of iterations, the standard deviation decreases nonlinearly, and the algorithm is characterized by deep excavation. This update is exactly the same as the value of plant growth for $r$ selection and k selection.

5. Competitive survival. If the current number of weeds and the number of seeds did not reach the preset maximum weed number $P_{\max }$, all weeds and seeds were used as the parent weed plants and returned to step 2 . If the current population exceeds the pre-set maximum weed number $P_{\max }$, the weeds and seeds in the population are arranged from large to small, with a pre- $P_{\text {max }}$ individual with a good fit Individuals are eliminated. Return to step 2 to repeat the calculation until the maximum iteration number reaches iter $r_{\max }$.

6. Output the best current weed and its fitness value, and come to the final solution to the problem.

\section{Establishment of Freight Location Assignment Model}

\subsection{Problem description and basic assumptions}

In this paper, the following warehouse model is established: There are a row of shelves in the warehouse, and each row of shelves has b column c layer. The nearest row of the warehouse entrance and exit is recorded as the first row, the column closest to the entrance of the shelf is recorded as the first column, and the bottom of the shelf is recorded as the first floor. A cargo position coordinate can be recorded as $(\mathrm{x}, \mathrm{y}, \mathrm{z})(\mathrm{x}=1, \ldots, \mathrm{a} ; \mathrm{y}=1, \ldots, \mathrm{b} ; \mathrm{z}=1, \ldots, \mathrm{c})$. The length of the unit cell is $L_{0}$; the unit mass of the $\mathrm{i}$-th product is $m_{i}$; the turnover rate of the $\mathrm{i}$-th product is $p_{i}$; the average moving speed in the $\mathrm{x}$-direction of the forklift is $v_{x}$; the average moving speed in the $\mathrm{y}$-direction of the forklift is $v_{y}$; $\mathrm{Z}$ direction of the average moving speed is $v_{z}$.

\subsection{Model analysis}

In this paper, the efficiency of the product into the library, similar products adjacent to the principle of shelf stability as a goal to optimize the establishment of multi-target location optimization mathematical model.

(1) the principle of similar products adjacent

Assuming that there are $\mathrm{n}$ pieces of goods in the warehouse, the coordinate vector of the i-type cargo space coordinates in the three-dimensional space is $\left[X_{i}, Y_{i}, Z_{i}\right]$, then the coordinate vector group of $n$ cargo spaces is:

$$
\left(\begin{array}{ccc}
x_{1} & y_{1} & z_{1} \\
x_{2} & y_{2} & z_{2} \\
\cdots & \cdots & \cdots \\
x_{n} & y_{n} & z_{n}
\end{array}\right)
$$

Set the i-type product location center coordinates vector: 


$$
R_{i}=\frac{1}{n} \sum_{i=1}^{n}\left[x_{i}, y_{i}, z_{i}\right]^{\prime}
$$

The resulting model is as follows:

$$
\min f_{1}=\sum_{x=1}^{a} \sum_{y=1}^{b} \sum_{z=1}^{c} \sqrt{\left(x-R_{i}(x)\right)^{2}+\left(y-R_{i}(y)\right)^{2}+\left(z-R_{i}(z)\right)^{2}}
$$

(2) the principle of out of storage efficiency

According to the product into the library frequency, the high turnover rate of products from the reservoir area near the place. The resulting model is as follows:

$$
\min f_{2}=\sum_{x=1}^{a} \sum_{y=1}^{b} \sum_{z=1}^{c}\left(\frac{x_{i}}{V_{x}}+\frac{y_{i}}{V_{y}}+\frac{z_{i}}{V_{z}}\right) \times L_{0} \times p_{i}
$$

(3) the principle of shelf stability

In order to ensure uniform load on the shelf, the quality of the product will be stored in the lower shelf, the model is as follows:

$$
\min f_{3}=\sum_{x=1}^{a} \frac{\sum_{y=1}^{b} \sum_{z=1}^{c} m_{i} \times n_{x y z i} \times z \times L_{0}}{\sum_{y=1}^{b} \sum_{z=1}^{c} m_{i} \times n_{x y z i}}
$$

Where $n_{x y z i}$ is the number of products that are packed in $\left(x_{i}, y_{i}, z_{i}\right)$ position.

\section{Algorithm implementation}

\subsection{Simulation parameter settings}

In this paper, the binary code is used to encode the cargo coordinates in the warehouse. It can be seen from the above model that the problem of cargo allocation is a multi-objective optimization problem. In this paper, we use the weighting coefficient method to transform the multi-objective optimization problem into single objective optimization problem.

$$
F(x, y, z)=\frac{1}{f(x, y, z)+1}=\frac{1}{w_{1} * \min f_{1}(x, y, z)+w_{2} * \min f_{2}(x, y, z)+w_{3} * \min f_{3}(x, y, z)+1}
$$

Where A, B, and C are as shown in equation (5) (6) (7).

The basic parameters of warehouse modeling are as follows:

The speed of the forklift: $v_{x}=1 \mathrm{~m} / \mathrm{s}, v_{y}=1 \mathrm{~m} / \mathrm{s}, v_{z}=0.5 \mathrm{~m} / \mathrm{s}$. Warehouse shelves have 6 rows and 6 columns 4 layers. There are three kinds of A.B.C products in the warehouse, the product quality is: $m_{A}=20 \mathrm{~kg}, m_{B}=10 \mathrm{~kg}, m_{C}=5 \mathrm{~kg}$. The product turnover rate is: $p_{A}=081, p_{B}=0.62$, $p_{C}=0.27$.

1. Determine the algorithm parameters.

The number of initial weeds $N_{0}$ is 30 , the maximum number of iter $r_{\max }$ is 100 , the maximum weed number $P_{\max }$ is 50, the maximum seed number $S_{\max }$ is 3 and the minimum seed number $S_{\min }$ is 1 , the non-linear modulation coefficient $\mathrm{n}$ is 3 , the initial standard deviation of weed diffusion $\sigma_{\text {initial }}$ is 2.5 and the final standard deviation $\sigma_{\text {final }}$ is 0.05 .

2. Initialize the weed position.

The location of the existing product in the warehouse is as follows:

Class A product storage location: $(2,2,1)(1,2,2)(2,3,1)(3,1,1)(1,1,1)(2,1,1)$;

Class B product storage location: $(5,4,1)(6,3,1)(6,3,2)(4,2,3)(5,3,1)(5,2,1)$;

Class C product storage location: $(2,6,1)(4,5,2)(3,3,1)(3,6,2)(3,5,1)(2,5,1)(3,5,2)$.

\subsection{Simulation results analysis}

The weed algorithm is used for the experimental operation according to the simulation parameters, and the simulation results are shown in Fig. 1. 


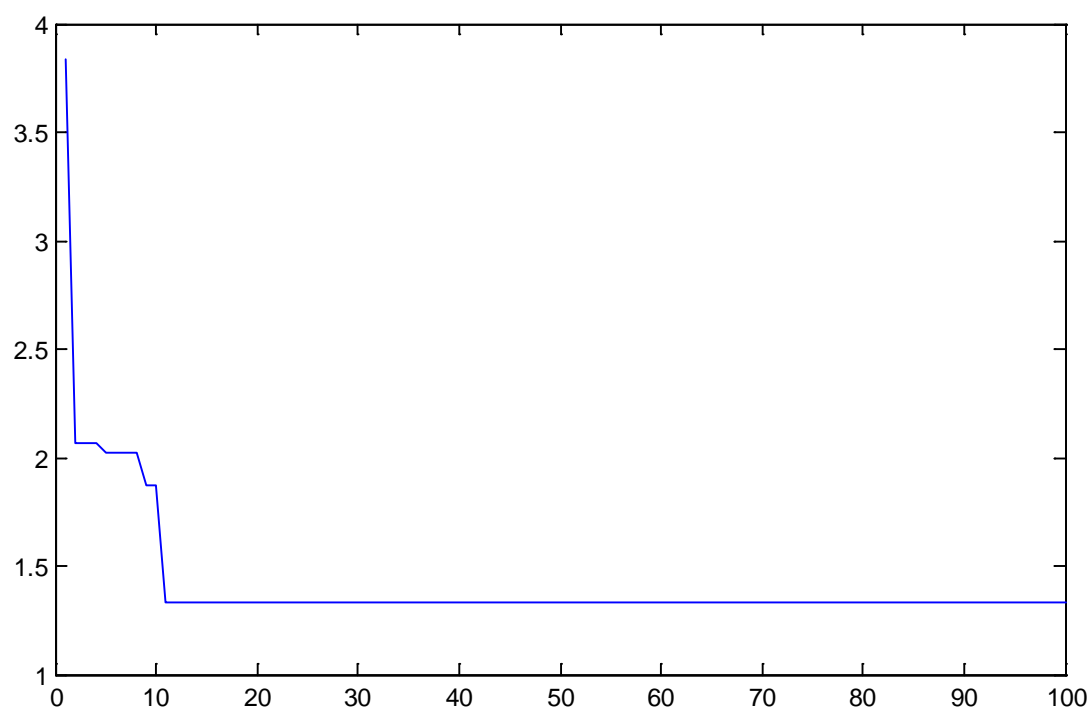

Fig. 1 The tracking graph of objective function solution

From the trend graph, we can see that the algorithm tends to be stable after the iteration of 10 generations, and the convergence is better.

\section{Conclusions}

This paper focuses on the optimization strategy of warehouse allocation of three-dimensional warehouse, and establishes a multi-objective optimization model for the optimal allocation of warehouse location. The IWO is used to solve the problem.

Through the simulation experiment and analysis, we can see that the IWO can effectively solve the problem of the distribution of the warehouse, improve the operation efficiency of the stacker, reduce the unnecessary workload and save the enterprise cost.

\section{Acknowledgments}

This work was supported by the National Science \& Technology Pillar Program during the 12th Five-year Plan Period (Grant No. 2015BAD18B05).

\section{References}

[1] AR Mehrabian, C Lucas, A novel numerical optimization algorithm inspired from weed colonization, Ecological Informatics. 2006, 1(4):355-366.

[2] S Karimkashi , AA Kishk, Invasive Weed Optimization and its Features in Electromagnetics, IEEE Transactions on Antennas \& Propagation. 2010, 58 (4):1269-1278.

[3] Semih Önüt, Umut R. Tuzkaya, Bilgehan Doğac. A particle swarm optimization algorithm for the multiple-level warehouse layout design problem [J]. Computers \& Industrial Engineering 2008,54 (4):783-799.

[4] Jinxiang Gu, Marc Goetschalckx, Leon F. Mc Ginnis. Research on warehouse operation: A comprehensive review[J]. European Journal of Operational Research 2007, (177):1-21.

[5] Changkyu Park,Junyong Seo. Comparing heuristic algorithms of the planar storage location assignment problem [J]. El SEVIER Annual Conference. Proceedings,2009.

[6] B. Muralidharan,Richard J. Linn,Ram Pandit. Shuffling heuristics for the storage location assignment in an AS/RS[J]. International Journal of Production Research, 1995,336.

[7] Sacramento Quintanilla,Ángeles Pérez,Francisco Ballestín,Pilar Lino. Heuristic algorithms for a storage location assignment problem in a chaotic warehouse[J].Engineering Optimization,2015,4710. 